\title{
Medial tibial stress syndrome: The relationship between gender and lower-extremity functional performance among collegiate track and field athletes
}

\author{
Megan E. Sievers BA, Andrew M. Busch EdD, CSCS \\ Ohio Wesleyan University.
}

\begin{abstract}
Medial tibial stress syndrome (MTSS) is a common lower extremity injury in track and field athletes. Many risk factors are associated with MTSS, and lower extremity performance may become altered in athletes suffering from prior symptoms, potentially increasing risk of future injury. The purpose of this study was to first examine the effect a prior history of MTSS has on lower-extremity measures, per gender, in collegiate level track and field athletes, and then determine if such measures predict future injury. Fifty-three healthy Division III collegiate track and field athletes (mean age $=19.40 \pm 1.13$ years) completed an injury history questionnaire along with five preseason lower-extremity functional tests including: ankle dorsiflexion (DF), single-leg anterior reach (SLAR), two timed single-leg balance (SLBAL) tests on a force plate, and single-leg hop for distance (SLH). Performance data were compared across gender and questionnaire data regarding injury history and occurrence of MTSS. Fifteen subjects $(28 \%)$ reported previous MTSS symptoms within the last 2 years. Chi-square analyses revealed females experienced more diagnoses compared to males $(p=.03)$. Independent $t$-tests revealed differences between gender on all SLBAL tests, as males performed better on all recorded measures $(p<.001-p$ $=.003$ ). No significant differences were noted in lower-extremity performance tests between subjects with and without prior MTSS injuries. Regression analyses using postseason injury questionnaire data revealed prior MTSS injuries had 17.3 higher odds of experiencing MTSS during the season (adjusted odds ratio $[A O R]=17.33,95 \% \mathrm{Cl}: 3.5-86.4 ; p=.001)$.

Keywords: Center-of-pressure excursion; Force plate; Movement system; Y-balance test; Track and field

${ }^{*}$ Correspondence to Author: Andrew M. Busch EdD, CSCS Ohio Wesleyan University

How to cite this article: Megan E. Sievers, Andrew M. Busch. Medial tibial stress syndrome: The relationship between gender and lower-extremity functional performance among collegiate track and field athletes. International Journal of Sports Medicine and Rehabilita-tion, 2021; 4:16.

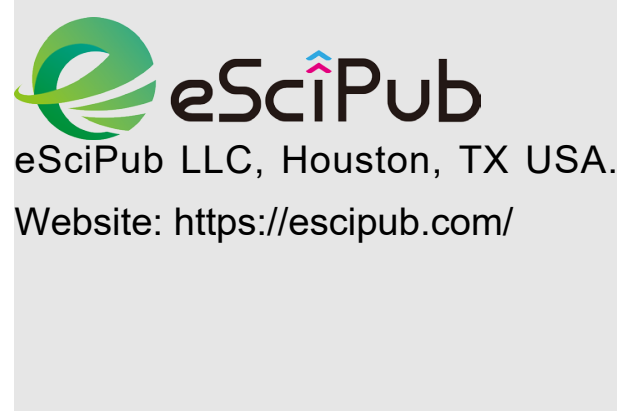




\section{INTRODUCTION}

Medial tibial stress syndrome (MTSS), commonly referred to as 'shin splints', is the most reported running-related musculoskeletal injury accounting for $13.6 \%-20 \%$ of all injuries sustained by runners ${ }^{[1]}$ and $60 \%$ of all overuse injuries in the lower extremities ${ }^{[2]}$. Due to the repetitive nature of running and impact loading of the lower extremities, the most common symptom is localized pain along the distal two-thirds of the posterior medial tibia ${ }^{[3,4]}$. The severity of symptoms can yield competitive time-loss ranging between a few practices to full competitive seasons, or even ending careers ${ }^{[5]}$. MTSS is frequently diagnosed in track and field athletes ${ }^{[6]}$, and 'shin splints' has historically been the generic label associated with any such exercise-induced pain localized in the medial tibia resulting from activities involving repeated foot-to-ground impact. However, the generality of the term shin splints creates ambiguity in origin and the location of pain, as exercise-induced lower leg pain can arise from mild overuse to more serious conditions ${ }^{[5]}$. It has been suggested to abandon the use of 'shin splints' and use MTSS instead, as it better describes the location and pathophysiology of the disorder [2,4,5,7-11]. MTSS differentiates clinically from several other terms surrounding exercise-induced lower leg pain including: tibial stress fractures [2,3,5,12,13], compartment syndrome ${ }^{[3,5,7,10,14]}$, periostitis ${ }^{[5,10,12,15]}$, and popliteal artery entrapment ${ }^{[3,5] \text {. }}$

\section{Risk Factors}

Over 100 different risk factors have been previously acknowledged with MTSS ${ }^{[4]}$. Several risk factors repeatedly showing strong associations with the development of MTSS or tibial stress fractures include: previous similar injuries ${ }^{[2,5,15-}$ ${ }^{17]}$, body mass index (BMI) ${ }^{[3,4,9]}$, navicular drop $[4,6,7,9-11,15,18,19]$, running technique $[5,11,14,20]$, hip internal and external ROM [4,9,15,18,21], plantar flexion strength $[2,5,15]$, ankle dorsiflexion ROM [2,5,19,22], and female gender [2,3,5,19,21,23]. Given the overall body of literature associated with different symptoms, we sought to further understand any residual impact previous MTSS diagnosis could have on several of the above mentioned risk factors, while including biomechanical and neuromuscular performance tasks in track and field athletes medically cleared to participate in a collegiate season. Several functional tests were chosen to quickly and easily evaluate the lower-extremity including: ankle dorsiflexion (DF) range of motion, single-leg anterior reach (SLAR), two single-leg balance (SLBAL) tests timed on a force plate, and a single-leg hop for distance (SLH).

Decreased ankle dorsiflexion (DF) has been noted by several authors as a risk factor for MTSS [2,5,19,22], however it is difficult to fully assess the role of DF as one meta-analysis found no significant evidence DF was associated with shin splints and its respective syndromes ${ }^{[9]}$. Such discrepancy could be due to the way DF was measured in different studies, as the weight bearing lunge method is believed to be more indicative of the actual DF available during functional activities ${ }^{[24-26]}$.

The SLAR test, performed as a modified version of the Y-Balance Test (YBT) (Functional Movement Systems ${ }^{\mathrm{TM}}$ Danville, VA), has been proven to be a reliable and efficient measure of dynamic balance and stability [27]. More specifically, asymmetries $>4 \mathrm{~cm}$ in anterior reaching tests have been found to be indicative of postural instability and increase the risk of lower extremity injury [28,29].

Single leg balance protocols assess postural stability and control, and the use of a force-plate captures sensitive information including: center of pressure (COP) excursion, medial-lateral (ML) velocity, and anterior-posterior (AP) velocity [30]. COP excursion represents the body's motion in space and total postural sway which helps quantify balance. Greater COP displacement, or increased postural sway, is associated with neuromuscular inefficiency ${ }^{[31]}$ and increased muscu-

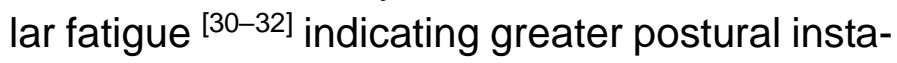
bility ${ }^{[30]}$. As lower leg muscles begin to fatigue when standing on a balancing device, a loss of stability is noticed when attempting to maintain equilibrium [ ${ }^{[1,32]}$. Both ML and AP velocities represent how quickly postural sway is occurring. The body's center of mass (COM) is anterior to 
the talocrural joint during quiet standing, and muscles surrounding the ankle play a key role in maintaining balance during SL stances as the plantar flexors specifically aid in postural corrections in the anteroposterior plane ${ }^{[32]}$. Corbeil et al. ${ }^{[31]}$ noted fatigue of these muscles increased postural sway and average velocities in both the $\mathrm{ML}$ and AP directions; suggesting the velocities increased as compensation for sensory and motor deficiencies created from peripheral muscular fatigue. While not commonly associated with MTSS, postural stability is important for maintaining proper gait and biomechanics during running. A lack of postural stability may lead to compensations such as overpronation and overstriding, both of which are implicated in MTSS. Greater balance and stability reduces ground-reaction forces (GRF) and postural sway in the ML direction which are factors shown to decrease lower extremity injury risk [33].

The SLH assessment is a unilateral test to assess functional movements in the lower extremity also required during running, while also assessing lower extremity strength and neuromuscular control ${ }^{[26,34]}$. It provides both clinical convenience and high reliability in healthy individuals and individuals after lower-extremity injuries [26], with the highest reliability among different hopping tests ${ }^{[35]}$. Brumitt et al. ${ }^{[34]}$ investigated several preseason screening assessments to identify collegiate athletes at risk for injury, and found significant associations in poor or asymmetric SLH performances with increased risk of lower extremity musculoskeletal injuries. When comparing both double-leg and SLH measures to identify lower-extremity performance deficits in individuals recovering from anterior cruciate ligament surgery, only SLH assessments were able to differentiate between healthy and recovering individuals ${ }^{[26]}$.

Therefore the purpose of this study was twofold; first to examine the effect a prior history of MTSS has on lower-extremity measures, per gender, in collegiate level track and field athletes, and furthermore determine if such measures predict future injury. It was hypothesized that athletes with a previous history of MTSS would have poorer performance on the lower-extremity tests, and therefore display a greater risk of developing MTSS throughout the season.

\section{MATERIALS and METHODS}

This study aimed to examine lower extremity differences among collegiate track and field athletes with and without a previous MTSS diagnosis, using five different functional tests. The tests were chosen due to their proven reliability in assessing the lower kinetic chain for functional movement. These measurements were time efficient, cost effective, and easy to administer in a limited space.

\section{Subjects}

National Collegiate Athletic Association (NCAA) Division III track and field athletes $(n=53$, age $=$ $19.40 \pm 1.13$ years) from a single university participated in this study. Among the subjects were 30 males, height $=70.6 \pm 2.24 \mathrm{~cm}$, weight $=$ $160.4 \pm 16.79 \mathrm{lbs}$.; and 23 females, height $=66.1$ $\pm 1.93 \mathrm{~cm}$, weight $=134.4 \pm 16.61 \mathrm{lbs}$. Inclusion criteria required athletes to be medically cleared for full participation in team practices and competitive events. Subjects were excluded if they were not listed on the active roster, reported a previous lower-extremity surgery, or reported a lower-extremity injury at the time of testing. All subjects provided written informed consent prior to testing, and the study was approved a university institutional review board: IRB Protocol \# 1902.006.

\section{Procedures}

All testing was completed two weeks before the start of the 2019-2020 indoor track and field season. Upon arrival at a university exercise laboratory, each subject was given an oral description of the study, and completed a demographic questionnaire indicating gender identification, age, athletic eligibility, dominant limb, main track events, and self-reported injury history. In regard to MTSS, subjects were asked if they had been diagnosed with MTSS by any medical professional (MD, ATC, PT, DC) on either leg in the past two years. Height was recorded to the nearest $\mathrm{cm}$, and mass to the nearest $0.1 \mathrm{~kg}$ using a standard stadiometer. Subjects then completed five different functional performance tests in the 
order of ankle dorsiflexion (DF), single-leg anterior reach (SLAR), two timed single-leg balance (SLBAL) tests on a force plate, and a single-leg hop for distance (SLH). After conclusion of the final indoor meet, subjects were asked to complete a survey to record MTSS injury occurrence during the season.

\section{Lower Extremity Assessments}

Ankle DF range of motion was assessed using a weight-bearing lunge method due to its practicality and high intra-rater reliability compared to non-weight bearing measures ${ }^{[24-26]}$. Subjects were instructed to remove their shoes, and using the wall as support, place their foot in line with a piece of tape located perpendicular with the wall.
They were instructed to bend their knee forward in line with their $2^{\text {nd }}$ toe, as far as possible, while keeping the heel of the tested foot in contact with the ground. The DF measurement was recorded using a digital inclinometer (model 82201B-00: Gain Express) and recorded to the nearest $0.01^{\circ}$ (Figure 1). This procedure was repeated twice for each leg with the average ROM of each leg used in statistical analysis. Prior to the main study, intraclass correlation coefficient estimates were calculated for DF on 10 individuals, resulting in an excellent intraclass correlation coefficient $(\mathrm{ICC}(3,1)=0.91, \mathrm{SEM}=2.63,95 \% \mathrm{Cl}=0.58$ 0.97).

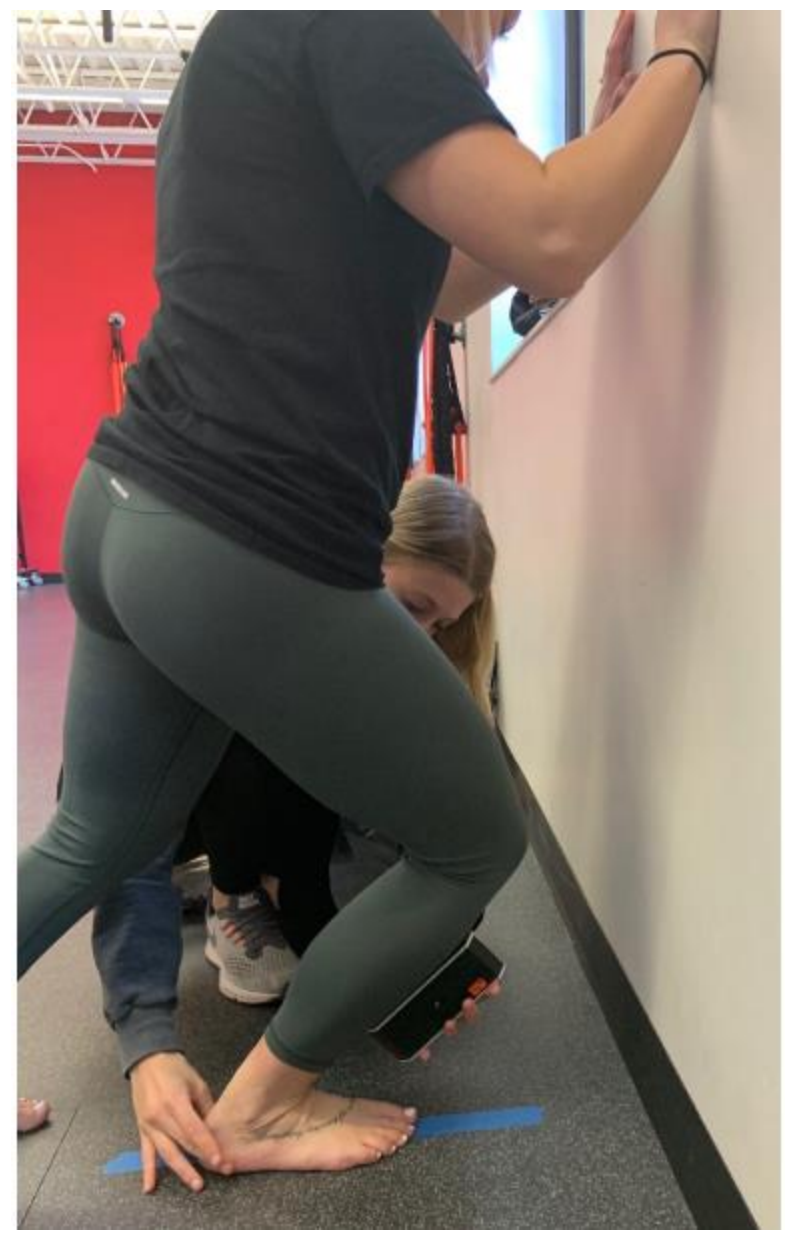

Figure 1: Dorsiflexion ROM positioning and administration using the weight-bearing lunge method.

The SLAR was performed using the anterior reach portion of the YBT as described by Plisky et al [27]. Subjects stood with shoes off as they performed the test, and were given two practice attempts with each foot, and for the test score to be recorded, they were instructed to remain in a single-leg stance the entire movement while avoiding kicking or stepping on the reach indicator. An examiner recorded two maximal-effort reach trials to the nearest $0.5 \mathrm{~cm}$. The best reach distance was normalized to limb length (\%LL), measured from the anterior superior iliac spine down to the medial malleolus, and used for statistical analysis (Figure 2). 


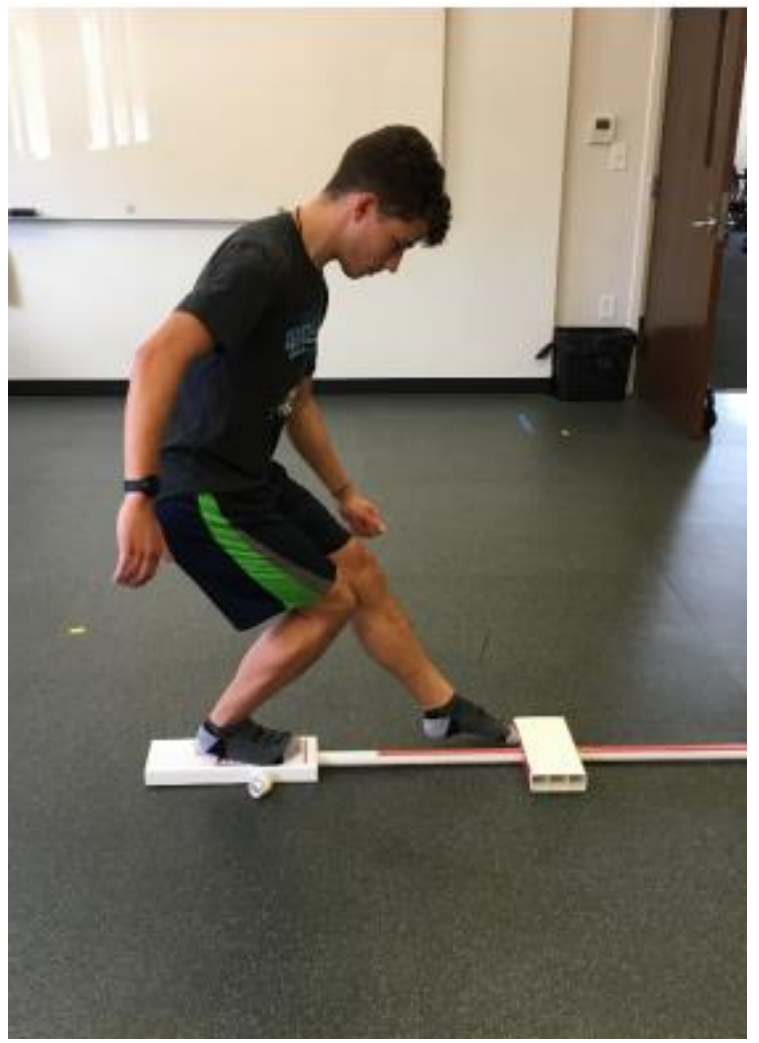

Figure 2: Single leg anterior reach using a Y-balance test kit.

Each SLBAL test was measured using a porta- before attempting the second test that mimicked ble force plate (FP5060 Bertec- Columbus, OH). the first test, with subjects standing barefoot on Subjects performed two 30-second SLBAL tests an Airex pad placed in the center of the force on each leg. They were instructed to stand bare- plate with eyes open. The middle 20 seconds of foot on the force plate with the knee fully ex- both tests were used for analysis, with the first tended on the tested leg, while the other leg was and last 5 seconds deleted to account for familheld comfortably off the ground with eyes closed iarization and fatigue.

(Figure 3). Data was recorded for both legs,

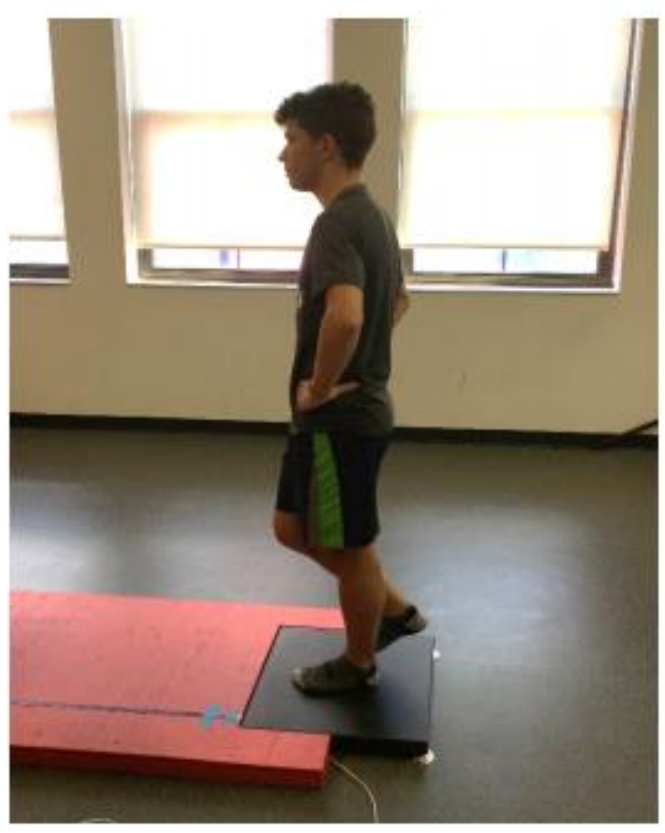

(a)

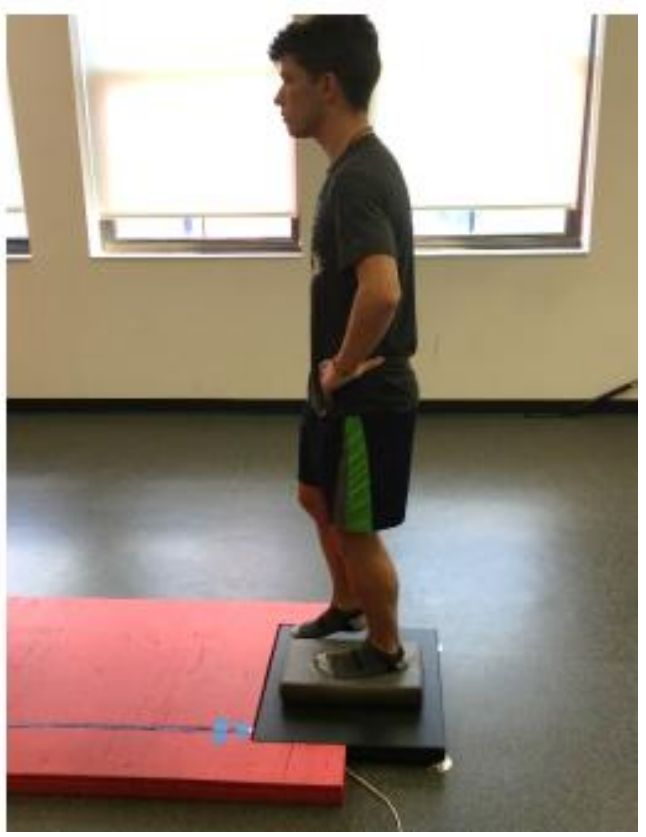

(b)

Figure 3: Single leg balance test barefoot (a) and on Airex pad (b). 
For the SLH, subjects were encouraged to wear shoes to minimize injury risk and pain upon landing. Subjects stood at the edge of the force plate, with a tape measure extended across a platform flush with the height of the force plate, to avoid jumping down off the force plate. Subjects were instructed to hop and land on the leg being tested, without aid from the other leg. They were given two practice jumps on each leg to familiarize themselves with the test trials. They also had to exhibit postural control for at least two seconds at landing before placing their other foot down for support (Figure 4). Trials were void if subjects landed with assistance from their opposite leg, lost balance, or took an extra step after landing. Distances were measured to the nearest $0.5 \mathrm{~cm}$ from the back heel of the jumping foot. Three successful trials were achieved on each leg with the best distance recorded for analysis.

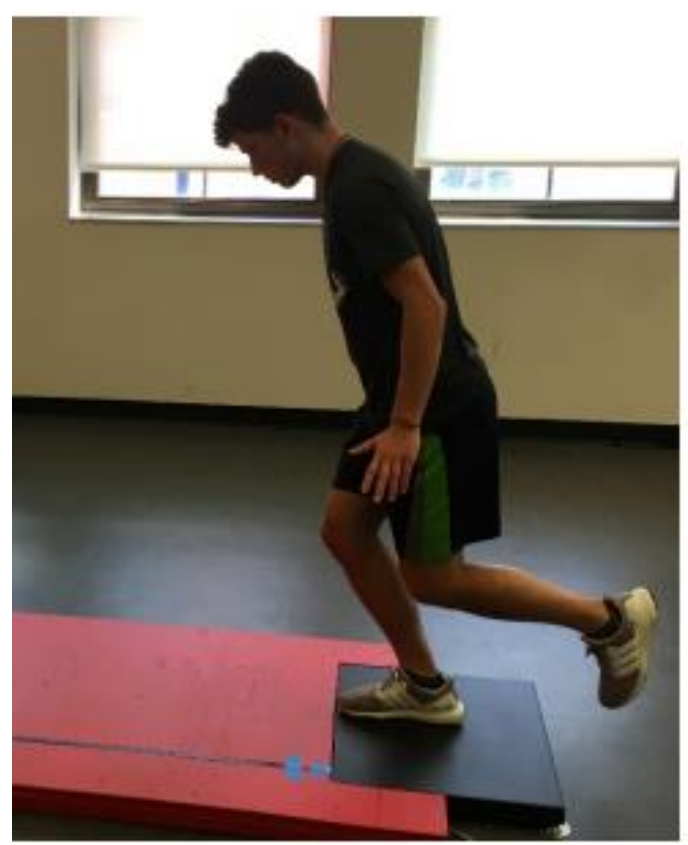

(a)

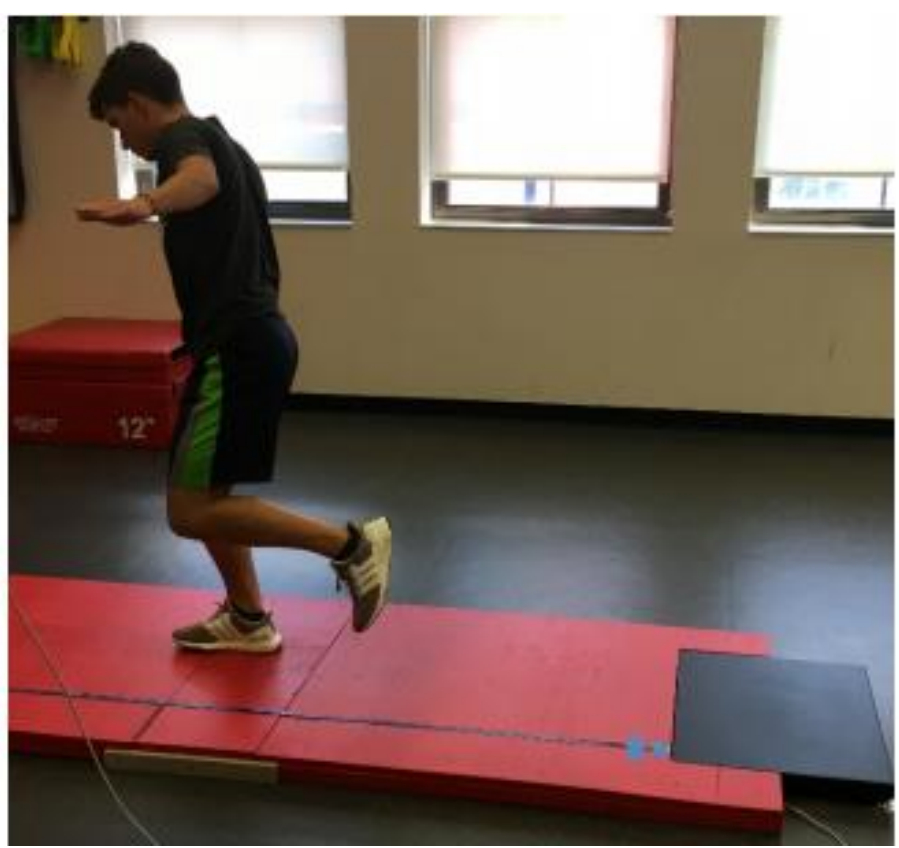

(b)

Figure 4: Single leg hop test starting position (a) and landing position (b).

\section{Statistical Analysis}

Data analyses were conducted using the Statistical Package for the Social Sciences version 26.0 (SPSS, Inc., Chicago, IL). Initial chi-square analyses were performed to assess relationships between gender and diagnosis of MTSS prior to the competitive season. Independent samples $t$-tests were used to assess the functional performance tests between gender and dominant vs. non-dominant legs. Bonferroni corrections were utilized to reduce the chance of Type I error in highly correlated dependent variables. A binomial logistic regression analysis was performed to determine effects of previous diagnosis of MTSS, gender, age, height, weight, balance, and dominant leg on the odds of being diagnosed during the season. Statistical significance was determined a priori at $p<.05$. Effect sizes were interpreted as very small $(<0.2)$, small (0.2-0.5), medium (0.5-0.8), or large $(>0.8)$ [36].

\section{RESULTS}

Distribution of the 53 subjects by main events were: 16 field event athletes (pole vault, long jump, triple jump, high jump), 4 sprinters $(100 \mathrm{~m}$, $200 \mathrm{~m}), 17$ mid-distance runners $(400 \mathrm{~m}, 800 \mathrm{~m})$, and 16 distance runners $(1500 \mathrm{~m}+)$. Initial injury questionnaire data revealed previous MTSS diagnosis was reported by 15 (28\%) subjects within the last two years (males $=5$, females $=$ 10). Chi-square analyses demonstrated a statistically significant association between gender and an diagnosis of MTSS, $x^{2}(1)=4.612, p=$ .032 , with a medium strength of association, $\varphi=$ $.295, p=.032$ (Figure 5). After a Bonferroni correction, the acceptable $p$-value for the 
independent samples $t$-tests was changed to $p<$ 0.0042 , revealing significant differences in all SLBAL measures between males and females. Males performed significantly better in all variables tested barefoot on the force plate (COP excursion, ML velocity, AP velocity) and standing on the Airex pad (COP excursion, ML velocity, AP velocity). Average measures and mean differences with $95 \%$ confidence intervals can be found in Table 1. The Cohen's effect sizes for all balance measures were large $(>0.8)$, with post hoc power analysis ranging between 0.92-0.99 for all measures, suggesting a high clinical significance between genders. No significant differences were noted in ankle DF, SLAR, SLH, or SLBAL measures between healthy subjects and those previously diagnosed with MTSS.

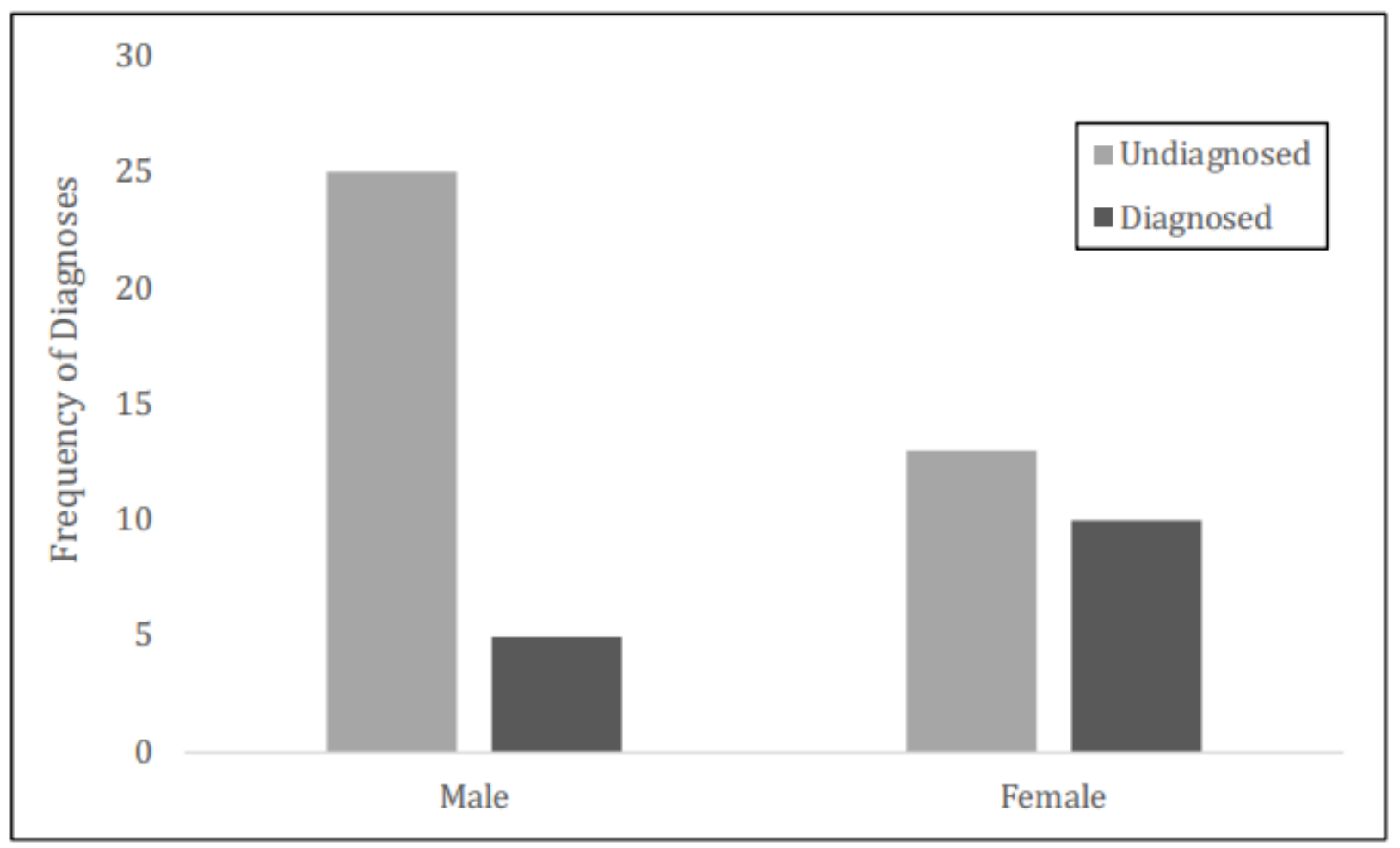

Figure 5: Frequency of pre-season MTSS diagnoses within the past 2-years by gender.

Postseason regression analysis only included 44 subjects who completed the postseason injury questionnaire. Of those who did not complete the questionnaire, four left the team for non-injury related reasons and five were unable to be reached for completion of the form. Data revealed $15(34 \%)$ subjects were diagnosed with MTSS at some point during the season. Binomial logistic regression revealed a statistically significant model $X^{2}(1, N=44)=15.03, p<.001$, explaining $40 \%$ of variance in being diagnosed and correctly classifying $81.8 \%$ of cases. Post hoc analysis was conducted, and revealed an achieved critical $z=1.96$, and power of 0.99 . Of the predictor variables, only previous diagnosis was statistically significant, whereas those with previous diagnosis of MTSS had a 17.3 fold greater likelihood of developing MTSS diagnosis during the season compared to those with no history of MTSS diagnosis (adjusted odds ratio $[\mathrm{AOR}]=17.33,95 \% \mathrm{Cl}: 3.5-86.4 ; p=.001$ ). With the observed AOR, post-hoc analysis demonstrated an achieved power of 0.99 .

\section{DISCUSSION}

Results from this study present several key findings. Initial data collection revealed significantly more females had been previously diagnosed with MTSS than males within the past two years. It is unknown however why this difference exists between genders. Whether the distinctions are structural in nature or caused from nutritional, genetic, or bone mass differences is unclear. It is widely accepted that females present with a greater Q-angle, yet no associations have been 
found when examining Q-angle as a risk factor for MTSS ${ }^{[9,19]}$. Bone density could also be related to higher female MTSS diagnoses. Lower bone densities can increase susceptibility to bone injuries due to decreased bone mass through which loads can be distributed, and stress fractures have been found to occur more often in female athletes possibly due to this lower bone density ${ }^{[5]}$. Females often have lower bone mass than males due to both normal and dysfunctional menstruation ${ }^{[2]}$. This theory is supported by Thacker et al. ${ }^{[15]}$ who found low calcium intake among female athletes to be a risk factor for shin splints, which, when coupled with increased calcium excretion during menstrual disturbances, can lower overall bone densities
[2]. Interestingly, Korpelainen et al. ${ }^{[37]}$ did not find significant differences in mean bone density values between female athletes suffering multiple lower extremity stress fractures compared to controls. It is also possible that females simply sought out medical attention from athletic trainers and team doctors more readily than males. Due to the limits of the present study, only demographic data and the functional measures described were collected. While several authors consider the female gender as a risk factor $[2,3,5,19,21,23]$, more in-depth research about gender differences is needed to understand why females seem to have higher incidences of MTSS and associated injuries.

Table 1. Single-Leg Balance Measures for Dominant and Nondominant Legs (Mean [Standard Deviation])

\begin{tabular}{|c|c|c|c|c|c|c|c|c|}
\hline \multirow[b]{2}{*}{ Protocol } & \multirow[b]{2}{*}{ Measure } & \multirow[b]{2}{*}{ Leg } & \multicolumn{2}{|l|}{ Gender } & \multirow{2}{*}{$\begin{array}{l}\text { Mean } \\
\text { Difference }\end{array}$} & \multirow{2}{*}{$95 \% \mathrm{Cl}$} & \multirow{2}{*}{$\begin{array}{l}\text { Effect } \\
\text { Size }\end{array}$} & \multirow{2}{*}{$P$ Value } \\
\hline & & & Male & Female & & & & \\
\hline \multirow{6}{*}{$\begin{array}{l}30 \text {-sec- } \\
\text { onds Foot } \\
\text { on Force } \\
\text { Plate }\end{array}$} & COP Excur- & Dominant & $4.59(0.54)$ & $5.19(0.59)$ & 0.603 & $(.286, .920)$ & -1.07 & $<.001^{*}$ \\
\hline & sion & Nondominant & $4.61(0.53)$ & $5.22(0.62)$ & 0.617 & $(.298, .937)$ & -1.07 & $<.001^{*}$ \\
\hline & ML Velocity & Dominant & $.166(.019)$ & $.188(.022)$ & 0.022 & $(.010, .033)$ & -1.08 & $<.001^{*}$ \\
\hline & & Nondominant & $.167(.019)$ & $.189(.023)$ & 0.023 & $(.011, .034)$ & -1.06 & $<.001^{*}$ \\
\hline & AP Velocity & Dominant & $.128(.015)$ & $.145(.017)$ & 0.017 & $(.008, .026)$ & -1.07 & $<.001^{*}$ \\
\hline & & Nondominant & $.128(.014)$ & $.145(.017)$ & 0.017 & $(.008, .026)$ & -1.11 & $<.001^{*}$ \\
\hline \multirow{6}{*}{$\begin{array}{ll}30-\text { sec- } \\
\text { onds } & \text { Foot } \\
\text { on } & \text { Airex } \\
\text { Pad } & \end{array}$} & Excur- & Dominant & $4.72(0.53)$ & $5.25(0.61)$ & 0.528 & $(.209, .847)$ & -0.94 & $.002^{*}$ \\
\hline & sion & Nondominant & $4.69(0.53)$ & $5.26(0.66)$ & 0.566 & $(.236, .896)$ & -0.97 & $.001^{*}$ \\
\hline & ML Velocity & Dominant & $.169(.019)$ & $.189(.022)$ & 0.02 & $(.008, .031)$ & -0.98 & $.001^{*}$ \\
\hline & & Nondominant & $.169(.019)$ & $.189(.023)$ & 0.021 & $(.009, .032)$ & -0.96 & $.001^{*}$ \\
\hline & AP Velocity & Dominant & $.132(.015)$ & $.146(.017)$ & 0.014 & $(.005, .023)$ & -0.88 & $.003^{*}$ \\
\hline & & Nondominant & $.130(.015)$ & $.146(.019)$ & 0.016 & $(.007, .025)$ & -0.95 & $.001^{*}$ \\
\hline
\end{tabular}

Note: $\mathrm{COP}=$ Center of Pressure, $\mathrm{ML}=$ Medial/Lateral, $\mathrm{AP}=$ Anterior/Posterior

Note: COP data are expressed as total distance traveled $(\mathrm{m})$

Note: $\mathrm{ML}$ and AP data are expressed as average velocity $(\mathrm{m} / \mathrm{sec})$

*Significant gender difference (Bonferroni Correction: $P<.0042$ )

Significant balance differences between genders were found for all SLBAL variables tested: COP, ML velocity, and AP velocity. For all tests barefoot on the force plate and while standing on an Airex pad, males performed significantly better than females, which is suggestive of greater stability and neuromuscular control ${ }^{[30,31]}$. This potentially could have implications for protection against lower extremity bone injury. One study investigating female athletes who completed dynamic balance training noted reductions in impact forces, GRF, and COP excursion in the ML direction, suggesting improved stability may help reduce the impact shock on the lower legs [33]. Elevated GRF have been found during faster tibial accelerations ${ }^{[37,38]}$ and heel striking ${ }^{[14,37]}$, which are thought to contribute to MTSS and incidence of stress fractures in the leg ${ }^{[20]}$. The current findings from this study may provide a link between lower leg stability and gender differences shown in the frequency of MTSS diagnoses. Females performed significantly worse on 
all balance measures, and with higher frequency of MTSS diagnoses. Further research on normative balance data is needed to provide greater understanding of these differences.

Disregarding gender, there were no associations found between subjects with a history of MTSS and those without regarding ankle DF, SLAR, SLBAL, or SLH measures, which does not support the authors' hypothesis. It is quite possible that with the given sample size $(n=53)$, there were not enough subjects or variation to find significant differences, as only 15 subjects reported previous MTSS diagnoses. It is also possible that subjects in the study may have refrained from discussing any shin pain with their coaches or athletic trainers for various reasons, including fear of losing training time, decreasing the overall amount of professional diagnoses. Two studies of high school and Division I collegiate athletes found the top three reasons athletes refrained from reporting concussive symptoms included: not wanting to lose playing time, not wanting to let their teammates down, and not

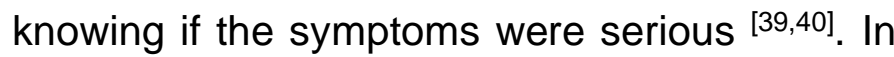
both studies, the top reason for withholding potential concussion information was to prevent the loss of playing time. The topic of self-reporting injuries is not prevalent in the literature, but it is plausible that subjects in the present study refrained from reporting MTSS or related injuries due to the above-mentioned reasons. Therefore, some subjects may have presented with reduced balance measures, but were not classified as having MTSS due to a lack of a diagnosis.

Both the SLAR and SLH measures were hypothesized to produce outcome differences between subjects with and without MTSS, due to their high reliabilities in identifying lower extremity musculoskeletal injuries ${ }^{[26,28,29]}$. Both tests were also predicted to identify limb asymmetries in subjects with MTSS, however none were found in the present study. It has previously been shown that YBT performance and non-contact injuries in Division I athletes across multiple sports, including track and field, have found asymmetry in the anterior direction increased the odds of injury [29]. However the results of the current study are similar to Ruffe et al. ${ }^{[41]}$ who found no associations of injuries with the anterior reach portion of the YBT. Brumitt et al. ${ }^{[34]}$ conducted a prospective cohort study examining 193 Division III college athletes across multiple sports to determine the ability of SLH performances at identifying risk for injury, and poor or asymmetric SLH performances were associated with an increased risk of lower extremity musculoskeletal injuries. The results of this study found no significant differences regarding limb asymmetries or poor performance in the lower extremity tests among those with prior MTSS diagnoses. This might be due to the inability of the tests to accurately measure factors related to the specific origin of subjects' MTSS injuries, and might be explained by the test's proficiency at identifying functional differences in parts of the lower extremity besides the tibia, including the foot and ankle, knee, or hip ${ }^{[26,34]}$. Since MTSS may arise from either skeletal $[2,29,30]$ or muscular $[2,18,33,37]$ factors, or a combination of both ${ }^{[2,5,9,12]}$, it is possible that the majority of injuries in those presenting with MTSS in the present study arose from problems within the tibia itself. In this case, the SLAR and SLH would not exhibit any performance differences, as a muscular component to the injury would not have existed. If possible, it may be beneficial to first determine the origin of pain before performing these tests so the most appropriate assessments could be implemented. Although it was not the goal of this study, more advanced technology such as magnetic resonance imaging or X-rays may help determine if the injury was muscular or skeletal in nature.

Previous diagnoses of MTSS were found to be the only significant predictor for injury recurrence, with those previously diagnosed within the past two years having a 17.3x higher likelihood of sustaining a future diagnosis. Of all variables tested, previous injury diagnosis was the only variable that produced significant retrospective results. This further supports previous research of running-related injuries showing large associations with injury recurrence, including MTSS, which may be due to potential alterations 
of gait biomechanics during running in order to protect the injured area ${ }^{[16]}$. If maintained for long periods of time, such alterations can become a new movement pattern leading to a heightened risk of re-injury. Secondly, if a bone is not given adequate time to heal after injury, repetitive stress will only exacerbate the injury further leading to an increased risk of injury recurrence ${ }^{[2,16]}$. Several other authors have noted previous injury magnifies the risk of sustaining similar future injuries ${ }^{[15,17]}$, suggesting previous occurrences of MTSS should be considered a risk factor for future MTSS, and since it can originate from a multitude of different injuries, coaches and athletic trainers should take previous MTSS diagnoses into account during preseason evaluations.

This study is not without limitations when interpreting the data. Subjects were selected from a single university which included a mixture of athletes who performed different running distances and field events. Injury history and MTSS diagnosis were self-reported by the athletes, and therefore could have been incorrectly reported. Examining athletes from multiple universities would provide greater diversity among subjects and may improve accuracy in outcome measures while strengthening associations between variables. Strength and conditioning practices were not investigated in terms of common exercises, or lack-thereof, which could play an important part in contributing to muscle imbalances in the lower extremity. Running volumes encountered during the season were also not recorded, which have been associated as risk factors for overuse injuries such as MTSS.

Further research is needed to assess the true influence of SLBAL on MTSS development. This study found potential links between balance performance and MTSS diagnoses with gender, but the two variables were not directly associated themselves. While no significance was found between MTSS and balance directly, balance should be included in MTSS screenings due to its proven relationship with GRF, impact forces, and reduced COP excursion. Gender, previous injury, and SLBAL should be considered when attempting to identify those more at risk for developing MTSS. Athletes who meet the at-risk criteria should be monitored closely throughout the season in order to catch early signs of injury that might otherwise go unnoticed.

\section{CONCLUSIONS}

This study attempted to identify relationships among athletes with previous MTSS diagnoses using five lower-extremity functional tests in Division III track and field athletes. Females had significantly more previous MTSS diagnoses while exhibiting significant balance deficits compared to males. Subjects with a history of MTSS were not found to differ in ankle DF, SLAR, SLBAL, or SLH performances when compared to those with no history of MTSS. Previous MTSS diagnosis was found to significantly increase the chances of re-injury by a factor of 17.3. This data extends knowledge of the effects of previous injury on future injury and potential effects of gender on the development of MTSS.

\section{PRACTICAL APPLICATIONS}

Based on these results, track and field coaches, athletic trainers, and physical therapists should assess athletes for previous MTSS injuries, as previous injury was the biggest predictor of future MTSS injuries. Females may be at a greater risk of sustaining MTSS running-related injuries, and single leg balance may be poorer in female track and field athletes compared to males.

\section{ACKNOWLEDGEMENTS}

The authors would like to thank the university for their support and allowing access to their athletes. The authors of this article have any conflicts of interest or financial conflicts to report. This research did not receive any specific grant funding agencies in the public, commercial, or not-for-profit sectors.

\section{REFERENCES}

[1] Lopes AD, Junior LCH, Yeung SS, Costa LOP. What are the Main Running-Related Musculoskeletal Injuries? Sports Med. Published online 2012:15.

[2] Couture CJ, Karlson KA. Tibial stress injuries: decisive diagnosis and treatment of "shin splints." The Physician and sportsmedicine. 2002;30(6): 29-36. doi:10.3810/psm.2002.06.337 
[3] Newman P, Witchalls J, Waddington G, Adams R. Risk factors associated with medial tibial stress syndrome in runners: a systematic review and meta-analysis. Open Access Journal of Sports Medicine. Published online November 2013:229. doi:10.2147/OAJSM.S39331

[4] Winkelmann ZK, Anderson D, Games KE, Eberman LE. Risk Factors for Medial Tibial Stress Syndrome in Active Individuals: An EvidenceBased Review. Journal of athletic training. 2016;51(12): 1049-1052. doi:10.4085/1062-60 50-51.12.13

[5] Beck BR. Tibial Stress Injuries: An Aetiological Review for the Purposes of Guiding Management. Sports Medicine. 1998;26(4):265-279. doi:10.216 5/00007256-199826040-00005

[6] Willems TM, De Clercq D, Delbaere K, Vanderstraeten G, De Cock A, Witvrouw E. A prospective study of gait related risk factors for exerciserelated lower leg pain. Gait \& Posture. 2006; 23(1): 91-98. doi:10.1016/j.gaitpost.2004.12.00 4

[7] Bates P. Shin splints--a literature review. British Journal of Sports Medicine. 1985;19(3):132137. doi:10.1136/bjsm.19.3.132

[8] Carr K, Sevetson E. How can you help athletes prevent and treat shin splints? (Cover story). Journal of Family Practice. 2008;57(6):406-408.

[9] Hamstra-Wright KL, Bliven KCH, Bay C. Risk factors for medial tibial stress syndrome in physically active individuals such as runners and military personnel: a systematic review and metaanalysis. Br J Sports Med. 2015;49(6):362-369. doi:10.1136/ bjsports-2014-093462

[10] Kortebein PM, Kaufman KR, Basford JR, Stuart MJ. Medial tibial stress syndrome. Medicine \& Science in Sports \& Exercise. 2000;32:S27.

[11] Kudo S, Hatanaka Y. Forefoot flexibility and medial tibial stress syndrome. Journal of orthopaedic surgery (Hong Kong). 2015;23(3):357-360.

[12] Vtasalo JT, Kvist M. Some biomechanical aspects of the foot and ankle in athletes with and without shin splints. The American Journal of Sports Medicine. 1983;11(3):125-130. doi:10.11 77/03635465 8301100304

[13] Brukner PD, Bennell KL. Review on Stress Fractures. Critical Reviews in Physical \& Rehabilitation Medicine. 2017;29(1-4):143-187. doi:10.16 15/critrevphysrehabilmed.v29.i1-4.40

[14] Diebal AR, Gregory R, Alitz C, Gerber JP. Effects of forefoot running on chronic exertional compartment syndrome: a case series. International journal of sports physical therapy. 2011;6(4):312-321.

[15] Thacker SB, Gilchrist J, Stroup DF, Kimsey CD. The prevention of shin splints in sports: a systematic review of literature. Medicine and science in sports and exercise. 2002;34(1):32-40.

[16] Hespanhol Junior LC, Pena Costa LO, Lopes AD. Previous injuries and some training characteristics predict running-related injuries in recreational runners: a prospective cohort study. Journal of Physiotherapy. 2013;59(4):263-269. doi:10.1016/S18 36-9553(13)70203-0

[17] Sanderlin BW, Raspa RF. Common stress fractures. American family physician. 2003;68(8): 1527

-1532 .

[18] McKeag DB, Dolan C, Garrick JG. Overuse Syndromes of the Lower Extremity. The Physician and sportsmedicine. 1989;17(7):108-123. doi:10. 1080/ 00913847.1989.11709830

[19] Messier SP, Martin DF, Mihalko SL, et al. A 2Year Prospective Cohort Study of Overuse Running Injuries: The Runners and Injury Longitudinal Study (TRAILS). The American journal of sports medicine. 2018;46(9):2211-2221. doi:10. 1177/03635 46518773755

[20] Hryvniak D, Dicharry J, Wilder R. Barefoot running survey: Evidence from the field. Journal of Sport \& Health Science. 2014;3(2):131-136.

[21] Buist I, Bredeweg SW, Lemmink KAPM, van Mechelen W, Diercks RL. Predictors of RunningRelated Injuries in Novice Runners Enrolled in a Systematic Training Program: A Prospective Cohort Study. The American Journal of Sports Medicine. 2010;38(2):273-280. doi:10.1177/ 036 3546509347985

[22] Fong C-M, Blackburn JT, Norcross MF, McGrath M, Padua DA. Ankle-dorsiflexion range of motion and landing biomechanics. Journal of athletic training. 2011;46(1):5-10. doi:10.4085/10 62-6050-46.1.5

[23] Grimston S k., Engsberg J r., Kloiber R, Hanley $D$ a. Menstrual, calcium and training history : relationship to bone health in female runners. Clinical Sports Medicine. 1990;2(3):119-128.

[24] Bennell KL, Talbot RC, Wajswelner H, Techovanich W, Kelly DH, Hall AJ. Intra-rater and inter-rater reliability of a weight-bearing lunge measure of ankle dorsiflexion. The Australian journal of physiotherapy. 1998;44(3):175-180.

[25] Konor MM, Morton S, Eckerson JM, Grindstaff TL. Reliability of three measures of ankle dorsiflexion range of motion. International journal of sports physical therapy. 2012;7(3):279-287.

[26] Onate JA, Starkel C, Clifton DR, et al. Normative Functional Performance Values in High School Athletes: The Functional Pre-Participation Evaluation Project. Journal of Athletic Training (Allen Press). 2018;53(1):35-42. doi:10.4085/1062$6050-458.16$ 
[27] Plisky PJ, Gorman PP, Butler RJ, Kiesel KB, Underwood FB, Elkins $B$. The reliability of an instrumented device for measuring components of the star excursion balance test. North American journal of sports physical therapy: NAJSPT. 2009; 4(2):92-99.

[28] Plisky PJ, Rauh MJ, Kaminski TW, Underwood FB.

Star Excursion Balance Test as a predictor of lower extremity injury in high school basketball players. The Journal of orthopaedic and sports physical therapy. 2006;36(12):911-919.

[29] Smith CA, Chimera NJ, Warren M. Association of $y$ balance test reach asymmetry and injury in division I athletes. Medicine and science in sports and exercise. 2015;47(1):136-141. doi:10. 1249/MSS. 0000000000000380

[30] Thompson LA, Badache M, Cale S, Behera L, Zhang N. Balance Performance as Observed by Center-of-Pressure Parameter Characteristics in Male Soccer Athletes and Non-Athletes. Sports (Basel, Switzerland). 2017;5(4). doi:10. 3390/ sports5040086

[31] Corbeil P, Blouin J-S, Bégin F, Nougier V, Teasdale N. Perturbation of the postural control system induced by muscular fatigue. Gait \& posture. 2003;18(2):92-100.

[32] Boyas S, Hajj M, Bilodeau M. Influence of ankle plantarflexor fatigue on postural sway, lower limb articular angles, and postural strategies during unipedal quiet standing. Gait \& posture. 2013; 37(4):547-551. doi:10.1016/j.gaitpost.20 12.09. 014

[33] Myer GD, Ford KR, Brent JL, Hewett TE. The effects of plyometric vs. dynamic stabilization and balance training on power, balance, and landing force in female athletes. Journal of strength and conditioning research. 2006;20(2):345-353.

[34] Brumitt J, Heiderscheit BC, Manske RC, Niemuth PE, Rauh MJ. Lower extremity functional tests and risk of injury in division iii collegiate athletes. International journal of sports physical therapy. 2013;8(3):216-227.

[35] Reid A, Birmingham TB, Stratford PW, Alcock GK, Giffin JR. Hop testing provides a reliable and valid outcome measure during rehabilitation after anterior cruciate ligament reconstruction. Physical Therapy. 2007;87(3):337-349. doi:10. 2522/ptj. 20060143

[36] Cohen J. Statistical Power Analysis for the Behavioral Sciences. 2nd ed. Lawrence Erlbaum Associates; 1988.

[37] 37. Korpelainen R, Orava S, Karpakka J, Siira $P$, Hulkko A. Risk factors for recurrent stress fractures in athletes. The American journal of sports medicine. 2001;29(3):304-310.
[38] Kreighbaum E, Barthels K. Biomechanics: $A$ Qualitative Approach for Studying Human Movement. 3rd Ed. Macmillan; 1990.

[39] Kerr ZY, Register-Mihalik JK, Kroshus E, Baugh $\mathrm{CM}$, Marshall SW. Motivations Associated With Nondisclosure of Self-Reported Concussions in Former Collegiate Athletes. The American journal of sports medicine. 2016;44(1):220-225. doi:10. 1177/0363546515612082

[40] Wallace J, Covassin T, Beidler E. Sex Differences in High School Athletes' Knowledge of Sport-Related Concussion Symptoms and Reporting Behaviors. Journal of athletic training. 2017;52(7): 682-688. doi:10.4085/1062-605052.3.06

[41] Ruffe NJ, Sorce SR, Rosenthal MD, Rauh MJ. Lower Quarter- and Upper Quarter Y Balance Tests as Predictors of Running-Related Injuries in High School Cross-Country Runners. International Journal of Sports Physical Therapy. 2019; 14(5): 695-706. doi:10.26603/ijspt201906 95 